\title{
An Approach and Implementation for Coupling Neurocognitive and Neuromechanical Models
}

\author{
Stephanie Goldfarb, Darren Earl, \\ and Vincent De Sapio \\ Information \& Systems Sciences Lab \\ HRL Laboratories, LLC \\ Malibu, California 90265-4797, USA \\ Email: \{segoldfarb, djearl, vdesapio\}@hrl.com
}

\author{
Misagh Mansouri and Jeffrey Reinbolt \\ Department of Mechanical, Aerospace, \\ \& Biomedical Engineering \\ The University of Tennessee \\ Knoxville, TN 37996-2210, USA \\ Email: \{misagh, reinbolt $\} @$ utk.edu
}

\begin{abstract}
The neuromechanics of human motion are generally represented in the literature by feedforward control mechanisms: the brain sends a control signal to a part of the body to move, and motion ensues. Thus neuromechanical commands for motion are influenced by control signals from neurocognitive inputs. However, feedback also exists from the neuromechanical system to the neurocognitive one, so that subsequent decisions related to motor commands are influenced by the motion itself. Recent work suggests that accounting for bidirectional feedback, both from neurocognitive to neuromechanical systems and from neuromechanical to neurocognitive ones, allows for more robust accounts of behavior. In this paper, we describe a neurocognitive model, a neuromechanical model, and a simple bidirectional feedback mechanism to couple the two systems. We then show that the behavior of the combined system is determined by the interaction of coupling strength and properties of the mechanical and cognitive models.
\end{abstract}

\section{INTRODUCTION}

This paper addresses coupled neurocognitive and neuromechanical control systems with applications to human-machine interfaces. Specifically addressed are generic coupled neurocognitive and neuromechanical models that can ultimately be tuned to demographic groups, individual subjects, and tasks. Work in modeling the interaction between decision-making and motor control is useful for the design and analysis of human-machine interfaces, where decisions are influenced by environmental stimuli and influence human motor behavior. This motor output acts on some machine interface (vehicle, robotic system, etc.) and accumulates evidence for subsequent decisions. A general coupled neuromechanical and neurocognitive system is shown in Figure 1.

Proper modeling of these coupled processes has application to a wide variety of human-machine systems including the design and incorporation of vehicle safety mechanisms, and the training of pilots through the identification of traits that make some pilots superior handlers of aircraft. Further, this work allows for improved human factors based design of machine interfaces by facilitating simulation based design and optimization of human-machine control interfaces using neurocognitive and neuromechanical models tuned to different behavioral phenotypes.

In this paper we present a method to couple neurocognitive and neuromechanical models. We consider in particular neurocognitive models describing the process of decision making

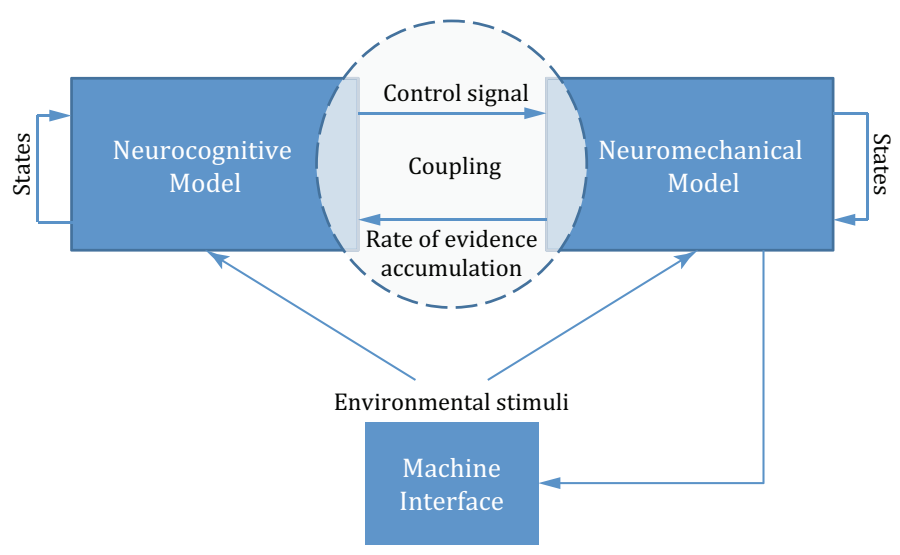

Fig. 1. Neuromechanical and neurocognitive models are coupled, such that ecisions are influenced by environmental stimuli and influence human motor behavior. This motor output acts on some machine interface (vehicle, robotic system, etc.) and the consequences of that action accumulate evidence for subsequent decisions.

leading up to and immediately following a decision to make a physical action or movement, and neuromechanical models describing the neural commands and muscle activations driving the motion itself.

While prior work has described such neurocognitive and neuromechanical models separately, the connections between these models have presented the neurocognitive processes as decisions directly preceding and enabling the neuromechanical processes. It has been shown that rather than a sequential process, internal neuromechanical processes are in fact influenced by neurocognitive processes both before and after a decision has been reached and that the coupling between neuromechanical and neurocognitive processes are responsible for a significant portion of behaviors in simple movements such as arm flexion [1]. However, an account of the nature of such coupling has not been provided. In this paper, we provide an account of how neuromechanical models and neurocognitive models may be coupled. We describe how a neurocognitive model may influence a neuromechanical model and vice versa, in real time.

Neurocognitive models serve as the basis for understanding human behavior in a range of tasks, for example discriminating 
motion direction, indicating a decision, and selecting among multiple alternatives in behavioral tasks. However, such models cannot alone account for corresponding neuromechanical processes. Similarly, neuromechanical models provide a basis for understanding human behavior, for example variations in gait, limb manipulation, and other examples of sensorimotor control. Inputs to both neuromechanical and neurocognitive models in these examples are from the environment. We describe a situation in which the models are coupled, so some of the inputs to the neuromechanical model are from the neurocognitive model, and vice versa.

The organization of this paper is as follows. In the following three sections, we first describe the neurocognitive model, then the neuromechanical model, and then a coupling scheme. The final section of this paper details our conclusions and directions for future work.

\section{Neurocognitive Model}

In this section we describe the neurocognitive decisionmaking model and then how this model may be tuned to account for different phenotypes of human behavior.

\section{A. Drift Diffusion Decision Making Model}

The neurocognitive model we use is a drift diffusion model (DDM) [2]. The DDM describes the noisy accumulation of evidence in favor of one alternative over another in two choice tasks, and the evolution of the DDM has been shown to correlate with both neurophysiology and behavior [3], [4]. The example addressed in this paper is that of a driver at a fourway stop. The decision is whether or not to make a left turn, as information (or stimuli) related to traffic in the other lanes is integrated regarding the safety of making the turn.

Evolution of the DDM is illustrated in Figure 2 and described by the equation

$$
d x=A d t+c d W
$$

in which information is accumulated from start point $x(0)=$ $x_{0}$ to one of two thresholds $\pm z$, and in which the drift (signal strength) is described by $A$, with Gaussian noise $c^{2}$ and Weiner process $d W$.

The utility of the DDM is due in part to its analytical simplicty. Closed form expressions for decision time (DT), or the time for accumulation of infromation to a threshold, is given by

$$
\begin{aligned}
\mathrm{DT}= & \frac{z}{A} \tanh \left(\frac{z A}{c^{2}}\right) \\
& +\frac{2 z}{A} \cdot\left(\frac{1-\exp \left(\frac{2 x_{0} A}{c^{2}}\right)}{\exp \left(\frac{2 z A}{c^{2}}\right)-\exp \left(-\frac{2 z A}{c^{2}}\right)}\right)-\frac{x_{0}}{A}
\end{aligned}
$$

The inaccuracy of a decision (e.g. deciding to turn when it is not safe to do so), or error rate (ER) is given by

$$
\mathrm{ER}=\frac{1}{1+\exp \left(\frac{2 z A}{c^{2}}\right)}-\left(\frac{1-\exp \left(\frac{2 x_{0} A}{c^{2}}\right)}{\exp \left(\frac{2 z A}{c^{2}}\right)-\exp \left(-\frac{2 z A}{c^{2}}\right)}\right)(3)
$$

Individual DDM parameters can be tuned to describe individual behavior.

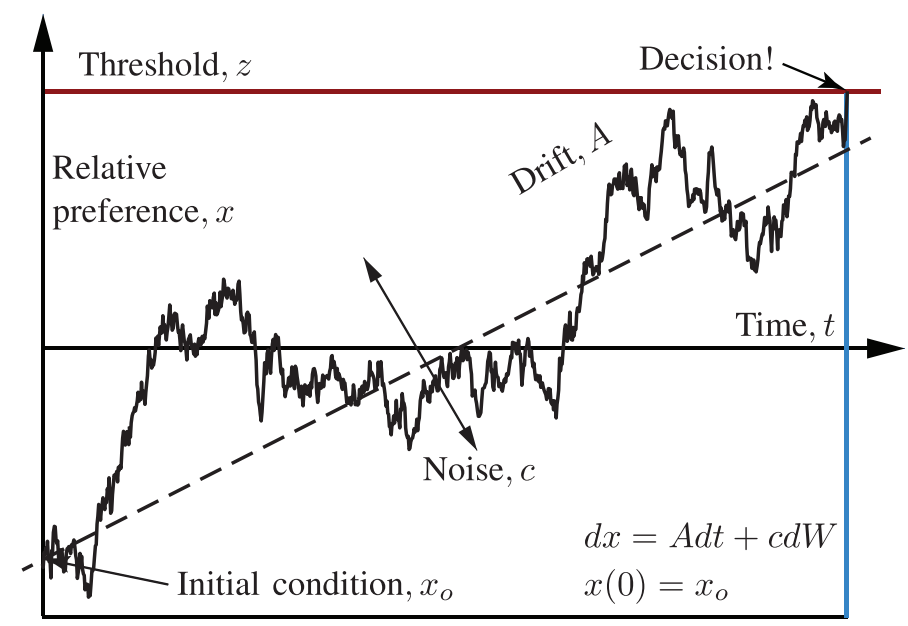

Fig. 2. The drift diffusion model describes the noisy accumulation of evidence from some initial condition $x_{0}$ to a threshold at $x(T)= \pm z$.

\section{B. Phenotypes and Tuning}

Individual differences in behavior attributed to differences in processing may be described by appropriate selection of parameters. Of particular interest to driver safety are these three phenotypes: conservative, aggressive, and distracted behavior in a left-hand turn task at a stop, illustrated in Figure 3. In each case we see the safety of the decision indicated in purple - at one second after reaching the stop, the situation changes and the turn becomes safe. The evolution of the decision variable is shown in yellow, and the decision threshold in light blue.

The conservative driver makes a decision accurately but slowly. His DT is long and noise is low, while the threshold is high. The distracted driver in this example does not make a decision to turn but rather remains stalled at the intersection, slowing the commutes of other drivers. In this case, noise is high. The aggressive driver in this example has a low threshold and a large initial condition, biased towards making the turn, even before it is safe to do so. When it becomes safe to make a turn, this driver is quick to turn.

\section{NeURomechanicAl Model}

The general neuromechanical model we present consists of muskoskeletal dynamics and neuromuscular dynamics, driven by a task space neuromuscular controller. We describe each of these components of the neuromechanical model below.

\section{A. Musculoskeletal Dynamics}

We begin by providing a description of the musculoskeletal plant. A general branching chain multibody system is described by the following system of $n$ equations in configuration space,

$$
\tau=\mathbf{M}(\mathbf{q}) \ddot{\mathbf{q}}+\mathbf{b}(\mathbf{q}, \dot{\mathbf{q}})+\mathbf{g}(\mathbf{q}),
$$

where $\mathbf{q} \in \mathbb{R}^{n}$ is the vector of generalized coordinates (joint angles), $\tau \in \mathbb{R}^{n}$ is the vector of generalized actuator forces (joint torques), $\mathbf{M}(\mathbf{q}) \in \mathbb{R}^{n \times n}$ is the configuration space mass matrix, $\mathbf{b}(\mathbf{q}, \dot{\mathbf{q}}) \in \mathbb{R}^{n}$ is the vector of centrifugal and Coriolis terms, and $\mathbf{g}(\mathbf{q}) \in \mathbb{R}^{n}$ is the vector of gravity terms. Equation (4) can be used to model musculoskeletal dynamics, 

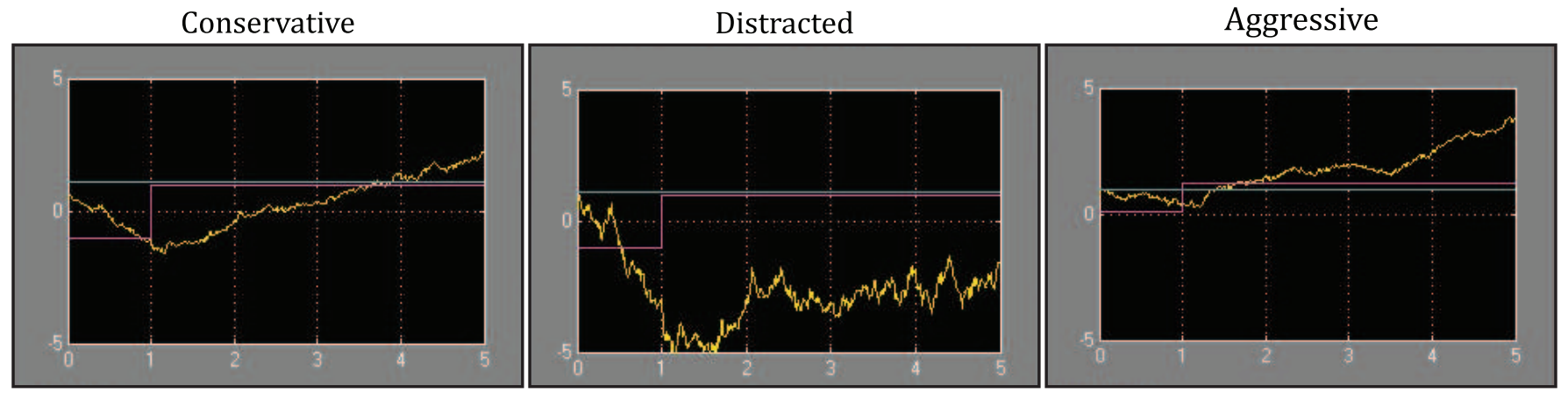

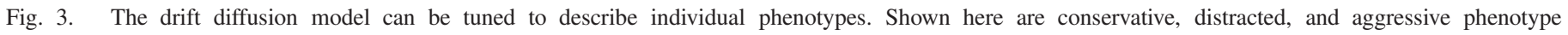
characterizations.

however, idealized torques at the joints, $\tau$, do not represent the mechanism by which the musculoskeletal system is actuated.

We now consider a set of $r$ musculo-tendon actuators spanning the skeletal system. We will assume that the vector of musculo-tendon lengths, $\mathbf{l} \in \mathbb{R}^{r}$, can be uniquely determined from the system configuration, $\mathbf{q}$. That is, $\mathbf{l}=\mathbf{l}(\mathbf{q})$. As a consequence of this assumption, differential variations in $\mathbf{l}$ are given by,

$$
\delta \mathbf{l}=\frac{\partial \mathbf{l}}{\partial \mathbf{q}} \delta \mathbf{q}=\mathbf{L}(\mathbf{q}) \delta \mathbf{q},
$$

where $\mathbf{L}(\mathbf{q}) \in \mathbb{R}^{r \times n}$ is the muscle Jacobian. From the principle of virtual work, we conclude that,

$$
\tau=-\mathbf{L}^{T} \mathbf{f}_{T}=\mathbf{R}(\mathbf{q}) \mathbf{f}_{T},
$$

where $\mathbf{f}_{T} \in \mathbb{R}^{r}$ is the vector of tendon forces. The negative sign is due to the convention of taking contractile muscle forces as positive. The matrix of moment arms is denoted $\mathbf{R}(\mathbf{q}) \in \mathbb{R}^{n \times r}$. From (4) and (6) the dynamics of the musculoskeletal system can be modeled as,

$$
\mathbf{R}(\mathbf{q}) \mathbf{f}_{T}=\mathbf{M}(\mathbf{q}) \ddot{\mathbf{q}}+\mathbf{b}(\mathbf{q}, \dot{\mathbf{q}})+\mathbf{g}(\mathbf{q}) .
$$

\section{B. Neuromuscular Dynamics}

The behavior of the musculo-tendon actuators can be modeled as a set of Hill-type active state force generating units [5], [6]. Activation dynamics refers to the process of muscle activation in response to neural excitation. This process can be modeled by the following equation of state written in terms of the vector of muscle activations, $\mathbf{a} \in \mathbb{R}^{r}$,

$$
\dot{\mathbf{a}}=\operatorname{diag}\left[\tau\left(u_{1}, a_{1}\right), \cdots, \tau\left(u_{r}, a_{r}\right)\right]^{-1}(\mathbf{u}-\mathbf{a}),
$$

where $a_{i} \in[0,1]$ and $u_{i} \in[0,1]$ is the neural input (excitation). The term $\tau\left(u_{i}, a_{i}\right)$ is a time constant given by,

$$
\tau\left(u_{i}, a_{i}\right)=\left\{\begin{array}{ll}
\left(\tau_{a}-\tau_{d}\right) u_{i}+\tau_{d}, & \text { if } u_{i} \geq a_{i} \\
\tau_{d}, & \text { if } u_{i}<a_{i}
\end{array},\right.
$$

where $\tau_{a}$ and $\tau_{d}$ are the activation and deactivation time constants, respectively.

Contraction dynamics refers to the process of force generation in the muscle based on muscle contraction, rate of contraction, and activation. This process can be modeled as a lumped parameter system which describes the configuration of forces. There is an active element, a passive viscoelastic element (in parallel), and an elastic tendon element (in series). The relative angle associated with the muscle fibers, $\alpha$, is referred to as the pennation angle.

We have the following relations,

$$
\begin{aligned}
& \mathbf{l}(\mathbf{q})=\mathbf{l}_{M} \cos (\alpha)+\mathbf{l}_{T}, \\
& \mathbf{i}(\mathbf{q})=\mathbf{i}_{M} \cos (\alpha)+\mathbf{l}_{T},
\end{aligned}
$$

where $\mathbf{l}_{M}$ is the vector of muscle lengths and $\mathbf{l}_{T}$ is the vector of tendon lengths. The following force equilibrium equation can be expressed,

$$
\mathbf{f}_{T}=\left(\mathbf{f}_{A}+\mathbf{f}_{P}\right) \cos (\alpha),
$$

where $\mathbf{f}_{A}$ is the vector of active forces in the muscles and $\mathbf{f}_{P}$ is the vector of passive forces in the muscles. Using this force equilibrium equation as well as constitutive relationships describing muscle forces as a function of muscle length and contraction velocity, $\mathbf{f}_{A}\left(\mathbf{l}_{M}, \mathbf{i}_{M}, \mathbf{a}\right)$ and $\mathbf{f}_{P}\left(\mathbf{l}_{M}, \dot{\mathbf{l}}_{M}\right)$, and tendon force as a function of tendon length, $\mathbf{f}_{T}\left(\mathbf{l}_{T}\right)$, we can express the following equation of state in functional form,

$$
\mathbf{i}_{M}=\mathbf{i}_{M}\left(\mathbf{l}(\mathbf{q}), \mathbf{i}(\mathbf{q}, \dot{\mathbf{q}}), \mathbf{l}_{M}, \mathbf{a}\right) .
$$

So, for a system of $r$ musculo-tendon actuators, we can express the following system of $2 r$ first-order state equations,

$$
\begin{aligned}
\dot{\mathbf{a}} & =\operatorname{diag}\left[\tau\left(u_{1}, a_{1}\right), \cdots, \tau\left(u_{r}, a_{r}\right)\right]^{-1}(\mathbf{u}-\mathbf{a}), \\
\dot{\mathbf{l}}_{M} & =\dot{\mathbf{l}}_{M}\left(\mathbf{l}(\mathbf{q}), \dot{\mathbf{l}}(\mathbf{q}, \dot{\mathbf{q}}), \mathbf{l}_{M}, \mathbf{a}\right),
\end{aligned}
$$

where the internal states are $\mathbf{l}_{M}$ and $\mathbf{a}$. The tendon force can be written in terms of the states as,

$$
\mathbf{f}_{T}=\mathbf{f}_{T}\left(\mathbf{l}(\mathbf{q}), \mathbf{l}_{M}\right) .
$$

\section{Task Space Neuromechanical Control}

Given the models described in the previous sections the states of the neuromechanical system are: $\mathbf{q}, \mathbf{v}, \mathbf{l}_{M}$, and $\mathbf{a}$, where the $2 n+2 r$ first order state equations consist of,

$$
\begin{aligned}
\dot{\mathbf{a}} & =\operatorname{diag}\left[\tau\left(u_{1}, a_{1}\right), \cdots, \tau\left(u_{r}, a_{r}\right)\right]^{-1}(\mathbf{u}-\mathbf{a}), \\
\dot{\mathrm{i}}_{M} & =\dot{\mathbf{l}}_{M}\left(\mathbf{l}(\mathbf{q}), \dot{\mathbf{l}}(\mathbf{q}, \dot{\mathbf{q}}), \mathbf{l}_{M}, \mathbf{a}\right), \\
\dot{\mathbf{q}} & =\dot{\mathbf{v}} \\
\dot{\mathbf{v}} & =\mathbf{M}(\mathbf{q})^{-1}\left[\mathbf{R}(\mathbf{q}) \mathbf{f}_{T}\left(\mathbf{l}(\mathbf{q}), \mathbf{l}_{M}\right)-\mathbf{b}(\mathbf{q}, \mathbf{v})-\mathbf{g}(\mathbf{q})\right] .
\end{aligned}
$$


More concisely the neuromechanical system can be represented as the following system of $2 n+2 r$ equations,

$$
\left(\begin{array}{cccc}
\dot{\mathbf{a}} & \mathbf{l}_{M} & \dot{\mathbf{q}} & \dot{\mathbf{v}}
\end{array}\right)^{T}=\mathbf{F}\left(\mathbf{a}, \mathbf{l}_{M}, \mathbf{q}, \mathbf{v}, \mathbf{u}\right) \text {. }
$$

A block diagram of this system is depicted in Figure 4.

Given a branching chain system and defining a set of $m$ task, or operational space, coordinates $\mathbf{x} \in \mathbb{R}^{m}$, we define the task Jacobian as,

$$
\mathbf{J}(\mathbf{q})=\frac{\partial \mathbf{x}}{\partial \mathbf{q}} \in \mathbb{R}^{m \times n} .
$$

These task space coordinates are related to the generalized coordinates by a functional mapping, and are chosen as a more natural space from which to formulate the control problem. As an example, they can be chosen as the Cartesian coordinates of some effector frame of the musculoskeletal system. The generalized actuator force or control torque, $\tau$, in (4) can then composed as $\mathbf{J}^{T} \mathbf{f}$, where $\mathbf{f} \in \mathbb{R}^{m}$ is the task, or operational space, force. In the redundant case an additional term needs to complement the task term in order to realize any arbitrary generalized force. We will refer to this term as the null space term and it can be composed as $\mathbf{N}^{T} \tau_{*}$, where $\tau_{*}$ is an arbitrary generalized force and $\mathbf{N}(\mathbf{q})^{T} \in \mathbb{R}^{n \times n}$ is the null space projection matrix. We then have the following set of task, or operational space, equations of motion [7],

$$
\mathbf{f}=\boldsymbol{\Lambda}(\mathbf{q}) \ddot{\mathbf{x}}+\mu(\mathbf{q}, \dot{\mathbf{q}})+\mathbf{p}(\mathbf{q}),
$$

where $\Lambda(\mathbf{q}) \in \mathbb{R}^{m \times m}$ is the operational space mass matrix, $\mu(\mathbf{q}, \dot{\mathbf{q}}) \in \mathbb{R}^{m}$ is the operational space centrifugal and Coriolis force vector, and $\mathbf{p}(\mathbf{q}) \in \mathbb{R}^{m}$ is the operational space gravity vector. These terms are given by,

$$
\begin{aligned}
\mathbf{\Lambda}(\mathbf{q}) & =\left(\mathbf{J M}^{-1} \mathbf{J}^{T}\right)^{-1} \\
\mu(\mathbf{q}, \dot{\mathbf{q}}) & =\mathbf{\Lambda} \mathbf{J} \mathbf{M}^{-1} \mathbf{b}-\mathbf{\Lambda} \dot{\mathbf{J}} \dot{\mathbf{q}} \\
\mathbf{p}(\mathbf{q}) & =\mathbf{\Lambda} \mathbf{J} \mathbf{M}^{-1} \mathbf{g} \\
\mathbf{N}(\mathbf{q})^{T} & =\mathbf{1}-\mathbf{J}^{T} \boldsymbol{\Lambda} \mathbf{J} \mathbf{M}^{-1}
\end{aligned}
$$

Thus, the overall dynamics of our multibody system, given by

$$
\tau=\mathbf{M} \ddot{\mathbf{q}}+\mathbf{b}+\mathbf{g},
$$

can be mapped into task space,

$$
\mathbf{M} \ddot{\mathbf{q}}+\mathbf{b}+\mathbf{g}=\tau \stackrel{\overline{\mathbf{J}}^{T}}{\rightarrow} \mathbf{f}=\mathbf{\Lambda} \ddot{\mathbf{x}}+\mu+\mathbf{p},
$$

where $\overline{\mathbf{J}}$ is the dynamically consistent inverse of the task Jacobian,

$$
\overline{\mathbf{J}}=\mathbf{M}^{-1} \mathbf{J}^{T} \boldsymbol{\Lambda} .
$$

The control torque can be decomposed into task and posture components,

$$
\tau=\underbrace{\mathbf{J}^{T} \mathbf{f}}_{\text {task component }}+\underbrace{\mathbf{N}^{T} \tau_{*}}_{\text {posture component }} .
$$

or expressed as,

$$
\tau=\mathbf{J}^{T}(\Lambda \ddot{\mathbf{x}}+\mu+\mathbf{p})+\mathbf{N}^{T} \tau_{*} .
$$

A controller employing (18) would be assumed to have imperfect knowledge of the system. Therefore, (18) should reflect estimates for the inertial and gravitational terms. Additionally, a control law needs to be incorporated. To this end we replace $\ddot{\mathbf{x}}$ in (18) with the input of the decoupled system, $\mathbf{f}^{\star}$ [8], to yield the dynamic compensation equation,

$$
\mathbf{f}=\widehat{\Lambda} \mathbf{f}^{\star}+\widehat{\mu}+\widehat{\mathbf{p}}
$$

where the $\widehat{.}$ represents estimates of the dynamic properties. Thus our control torque is,

$$
\tau=\mathbf{J}^{T}\left(\widehat{\mathbf{\Lambda}} \mathbf{f}^{\star}+\widehat{\mu}+\widehat{\mathbf{p}}\right)+\widehat{\mathbf{N}}^{T} \tau_{*} .
$$

We use (26) and (6) to express the muscle actuated control equation [9],

$$
\mathbf{R f}_{T}=\mathbf{J}^{T}\left(\widehat{\Lambda} \mathbf{f}^{\star}+\widehat{\mu}+\widehat{\mathbf{p}}\right)+\widehat{\mathbf{N}}^{T} \tau_{*} .
$$

Solving for $\mathbf{f}_{T}$ is typically an underdetermined problem, with more muscle actuators than generalized coordinates. In the computed muscle control (CMC) approach of [10] and [11] this actuator redundancy is resolved through a static optimization step. In our task-level formulation we also specify a static optimization step. Specifically we seek a set of muscle activations, ã, which minimize $\|\tilde{\mathbf{a}}\|^{2}$, where ã are muscle activations associated with the steady state tendon forces $\tilde{\mathbf{f}}_{T}(\tilde{\mathbf{a}})$ (i.e., tendon forces at a time when the contraction dynamics have equilibrated and the transients have decayed). Further these activations must satisfy the inequality constraints, $0 \leq a_{i} \leq 1$, and generate steady state tendon forces that satisfy,

$$
\mathbf{R} \tilde{\mathbf{f}}_{T}(\tilde{\mathbf{a}})=\mathbf{J}^{T}\left(\widehat{\Lambda} \mathbf{f}^{\star}+\widehat{\mu}+\widehat{\mathbf{p}}\right)+\widehat{\mathbf{N}}^{T} \tau_{*} .
$$

As in the joint space approach of [10] we can use a linear proportional controller to generate muscle excitations, $\mathbf{u}$, that cause the actual activations, a, from the forward neuromuscular simulation to track ã,

$$
\mathbf{u}=\tilde{\mathbf{a}}+\mathbf{K}_{a}(\tilde{\mathbf{a}}-\mathbf{a}),
$$

where $\mathbf{K}_{a}$ is a gain. This task-level computed muscle control architecture is depicted in Figure 5 with each of the functional modules highlighted. The quality of the internal model of the task space dynamics can be modulated by using imperfect or incomplete estimates of the task space properties. Other properties within the controller that can be modulated include the neuromuscular cost criterion, the task space tracking error (through the choice of control law, control gains, etc.), and the tracking error in the activation dynamics (through the choice of $\mathbf{K}_{a}$ ). Specific neuromechanical phenotypes can be generated based on tuning these motor control parameters as well as parameters in the musculoskeletal plant (Figure 4) such as maximum isometric muscle strengths, activation/deactivation time constants, and other properties that may reflect the effects of aging or musculoskeletal pathologies.

For our neuromechanical controller we can make the assumption that the tendons are infinitely stiff. In this case $l_{M}$ is no longer an independent state, but rather, it is algebraically related to the overall musculo-tendon length, $l(\mathbf{q})$. We can define the muscle saturation force, $f_{S}$, as the active muscle force at full activation $(a=1)$. So, $f_{S}(\mathbf{q}, \dot{\mathbf{q}}) \triangleq f_{A}(\mathbf{q}, \dot{\mathbf{q}}, 1)$. If we take the pennation angle, $\alpha$, and the passive force in the muscle, $f_{P}$, to be zero we have,

$$
\mathbf{f}_{T}=\mathbf{f}_{A}=\operatorname{diag}\left[f_{S_{1}}, \cdots, f_{S_{r}}\right] \mathbf{a}=\mathbf{K}_{f} \mathbf{a},
$$

where $\mathbf{K}_{f} \in \mathbb{R}^{r \times r}$ is the muscle force-activation transfer function, defined as the magnitude of force generation in the 


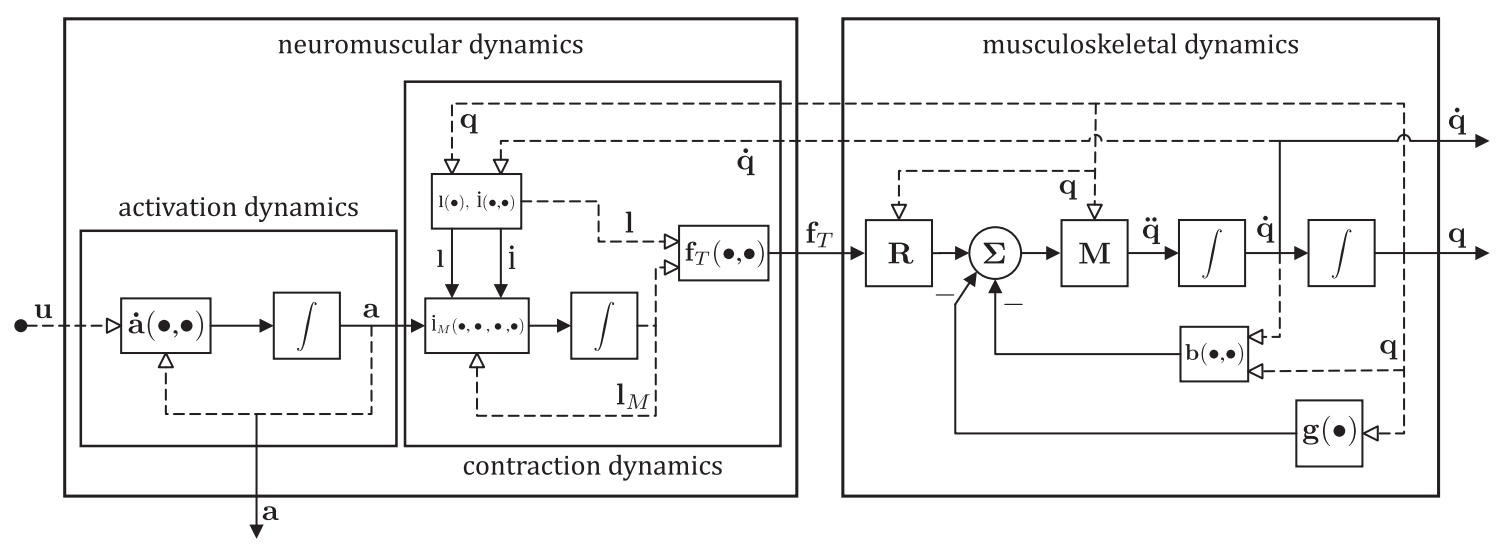

Fig. 4. Neuromuscular and musculoskeletal system (feed-forward path). Neural excitations provide input to the activation dynamics. Output of the activation dynamics provides input to the contraction dynamics. Output of the contraction dynamics provides input to the musculoskeletal dynamics through the tendon forces.

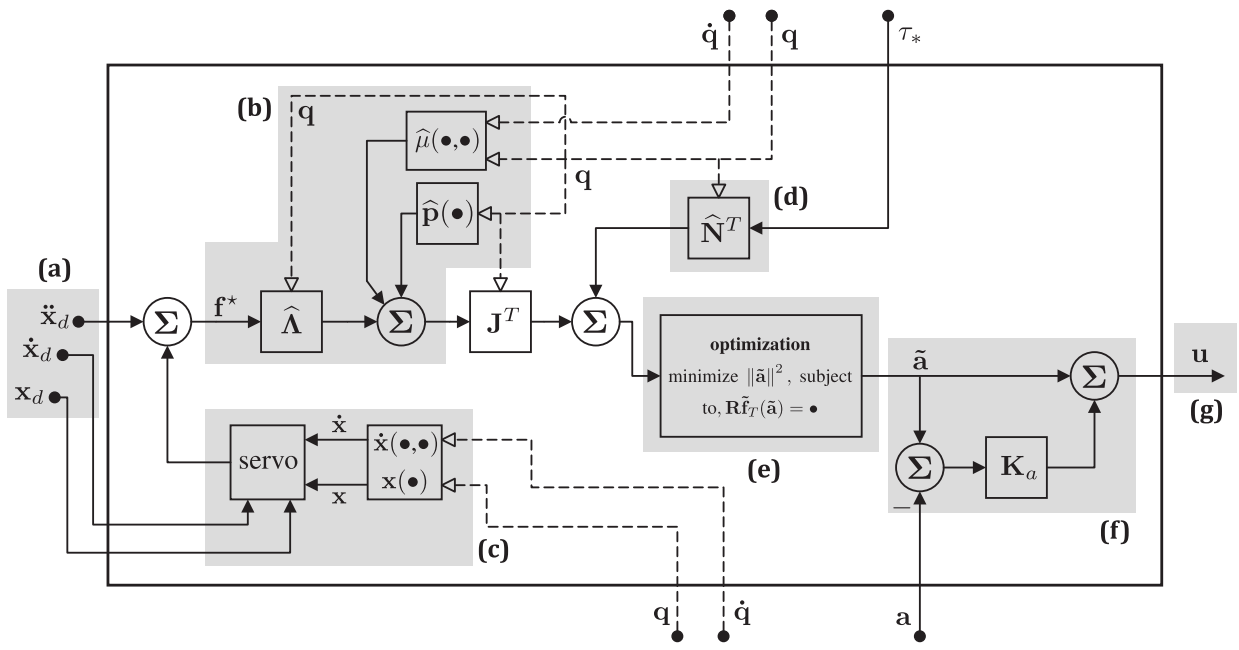

Fig. 5. A task-level neuromuscular controller based on a reformulation of the computed muscle control approach in task space. (a) Task-level command input. (b) Task space feedback linearization using task space inertial properties. (c) Task space feedback loop for tracking task space commands. (d) Posture space control input. (e) Neuromuscular cost optimization. (f) Activation feedback loop for tracking optimization solution. (g) Low-level motor output (neural excitations).

muscle per change in unit activation. The control equation, (28), can then be represented as,

$$
\mathbf{R K}_{f} \tilde{\mathbf{a}}=\mathbf{J}^{T}\left(\widehat{\mathbf{\Lambda}} \mathbf{f}^{\star}+\widehat{\mu}+\widehat{\mathbf{p}}\right)+\widehat{\mathbf{N}}^{T} \tau_{*} .
$$

A quadratic programming routine would need to be employed to solve the the minimization problem with equality and inequality constraints,

\section{Minimize $\|\tilde{\mathbf{a}}\|^{2}$}

Subject to the inequality constraints,

$0 \leq \tilde{a}_{i} \leq 1$,

and the equality constraints,

$$
\mathbf{R K}_{f} \tilde{\mathbf{a}}=\mathbf{J}^{T}\left(\widehat{\mathbf{\Lambda}} \mathbf{f}^{\star}+\widehat{\mu}+\widehat{\mathbf{p}}\right)+\widehat{\mathbf{N}}^{T} \tau_{*} .
$$

Specifying a linear (proportional) control law as the input of the decoupled system,

$$
\mathbf{f}^{\star}=\mathbf{K}_{x}\left(\mathbf{x}_{d}(t)-\mathbf{x}\right),
$$

not compensating for the task space musculoskeletal dynamics (using estimates of $\widehat{\Lambda}=\mathbf{1}, \widehat{\mu}=\mathbf{0}, \widehat{\mathbf{p}}=\mathbf{0}$ ), and omitting the null space control term simplifies our control to,

$$
\mathbf{R K}_{f} \tilde{\mathbf{a}}=\mathbf{J}^{T} \mathbf{K}_{x}\left(\mathbf{x}_{d}(t)-\mathbf{x}\right) .
$$

Figure 6 demonstrates this neuromechanical control methodology. Using a Matlab interface [12] to the OpenSim [13] musculoskeletal dynamics engine, a task command input (e.g., hand position) was specified. The hand was driven to three locations (pink spheres) while balancing a pole. Disturbance forces were applied to the pole (green arrow). The IPOPT library was used to solve the quadratic optimization problem. We have applied the same model methodology to account for an individual making a left hand turn while driving.

\section{Model Coupling}

In this section, we describe the method with which our neuromechanical and neurocognitive models are coupled. We then illustrate the influence of driver characterization and coupling strength on overall behavior in the simulation. 


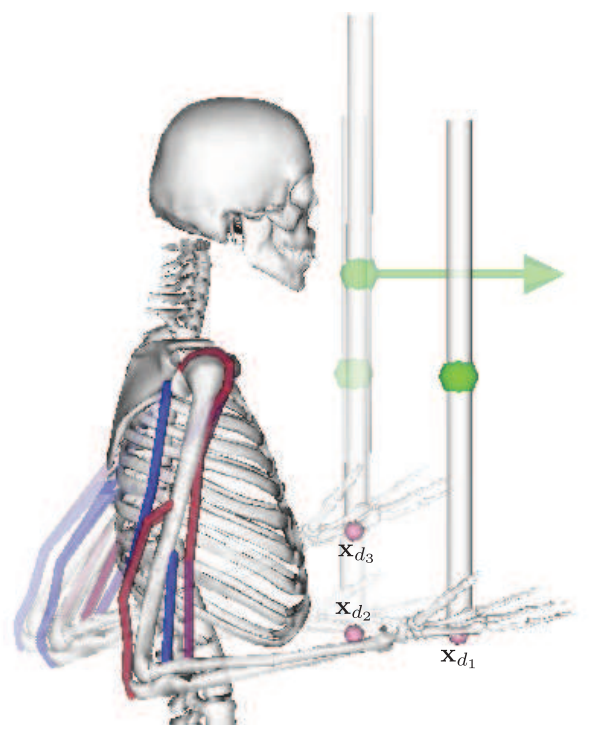

Fig. 6. Using a Matlab/Simulink interface [12] to the OpenSim [13] musculoskeletal dynamics engine, a task command input (e.g., hand position) was specified. The neuromechanical controller was implemented in Simulink and drives the hand driven to three locations (pink spheres) while balancing a pole. Disturbance forces were applied to the pole (green arrow). The IPOPT library was used to solve the quadratic optimization problem.

\section{A. Coupling Implementation}

To implement our model coupling, we include feedback from the neuromechanical model to the neurocognitive model, as well as feedback from the neurocognitive model to the neuromechanical one. The composite system is illustrated in Figure 1. The feedback from the neurocognitive to neuromechanical models is implemented via the control signal from the DDM: once a threshold accumulation level for the decision variable has been reached, a "go" signal is sent to the neuromechanical model.

The "go" signal consists of a truncated linear activation function of the decision variable from the DDM, and it consists of a desired position, sent to both shoulder and elbow, which can take on low, medium, or high values, corresponding to motion for the turn.

The feedback from the neuromechanical to the neurocognitive model consists of a contribution to the rate of evidence accumulation (drift rate) in the DDM: the difference of the sum of muscle activations for left and right turns are added to the baseline drift rate, so that the overall drift rate increases as the individual becomes more active and engaged in the turn.

\section{B. Coupling Simulation}

The coupling described above was implemented in Simulink and OpenSim for a simulation of driver behavior at a four way stop. Screenshots of the interface are shown in Figures 7 and 8 . Results of the coupling simulation are shown in Figures 9-10. In particular, we compare aggressive and conservative parameterizations with strong and weak coupling. In both figures, aggressive parameterizations are indicated with green lines, and conservative parameterizations are indicated with blue lines. Strong coupling is indicated by solid lines and weak coupling by dashed lines, such that a green solid line

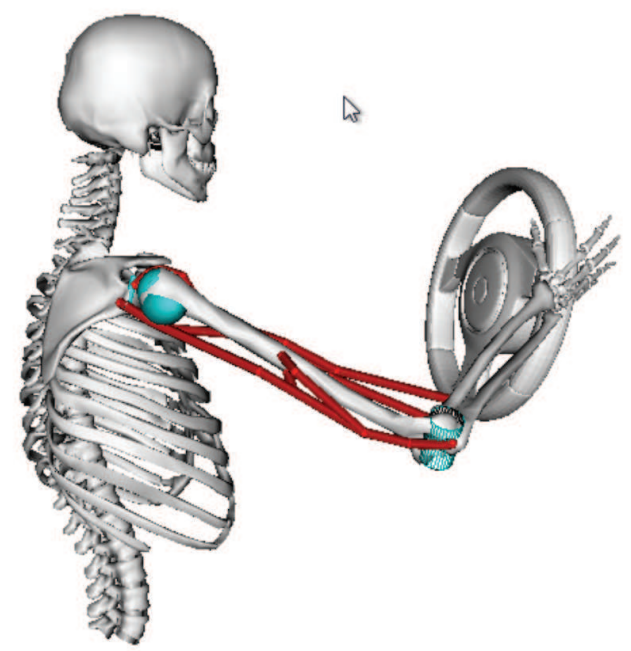

Fig. 8. OpenSim model of steering using the Simulink control interface. The driver makes a left turn based on the control signal from the DDM executed by the neuromechanical controller. The feedback from the neuromechanical model to the DDM consists of a contribution to the rate of evidence accumulation (drift rate) in the DDM.

indicates an aggressive driver with strong coupling. We see that both the strength of the coupling and the aggressiveness of the driver influence the behavior of the composite system.

Figure 9 shows the evolution of coupling variables over time. From the top, we see the drift variable, decision variable, and control signals to the shoulder and elbow, respectively. We see that the aggressive parameterization with strong coupling accumulates evidence at maximum intensity for nearly twice as long as the conservative parameterization with strong coupling. The difference in evidence accumulation leads to differences in the decision variables and corresponding control signals to the shoulder and elbow, with large values of the decision variable and control signals corresponding to more aggressive turn patterns.

Figure 10 shows comparative trajectories for the four systems over time. We see that the aggressive driver makes a turn with a wider swing, indicating less control of the vehicle. The swing width is moderated by coupling strength: for both aggressive and conservative drivers, strong coupling also leads to turns with wider swings than weak coupling.

\section{CONCLUSION}

In this paper, we proposed a simple coupling mechanism to describe the bidirectional feedback between neuromechanical and neurocognitive model systems. We then studied the influence of the model coupling on decision variables as well as on ultimate vehicle trajectory. We found that the strength of the coupling moderates the influence and role of phenotypes. In particular, we found that strong coupling leads to wider, less safe turns. Directions for future work include expanding upon the range of phenotypes and coupling schemes described and tested in our simulations and validating them against data collected from human subjects. 


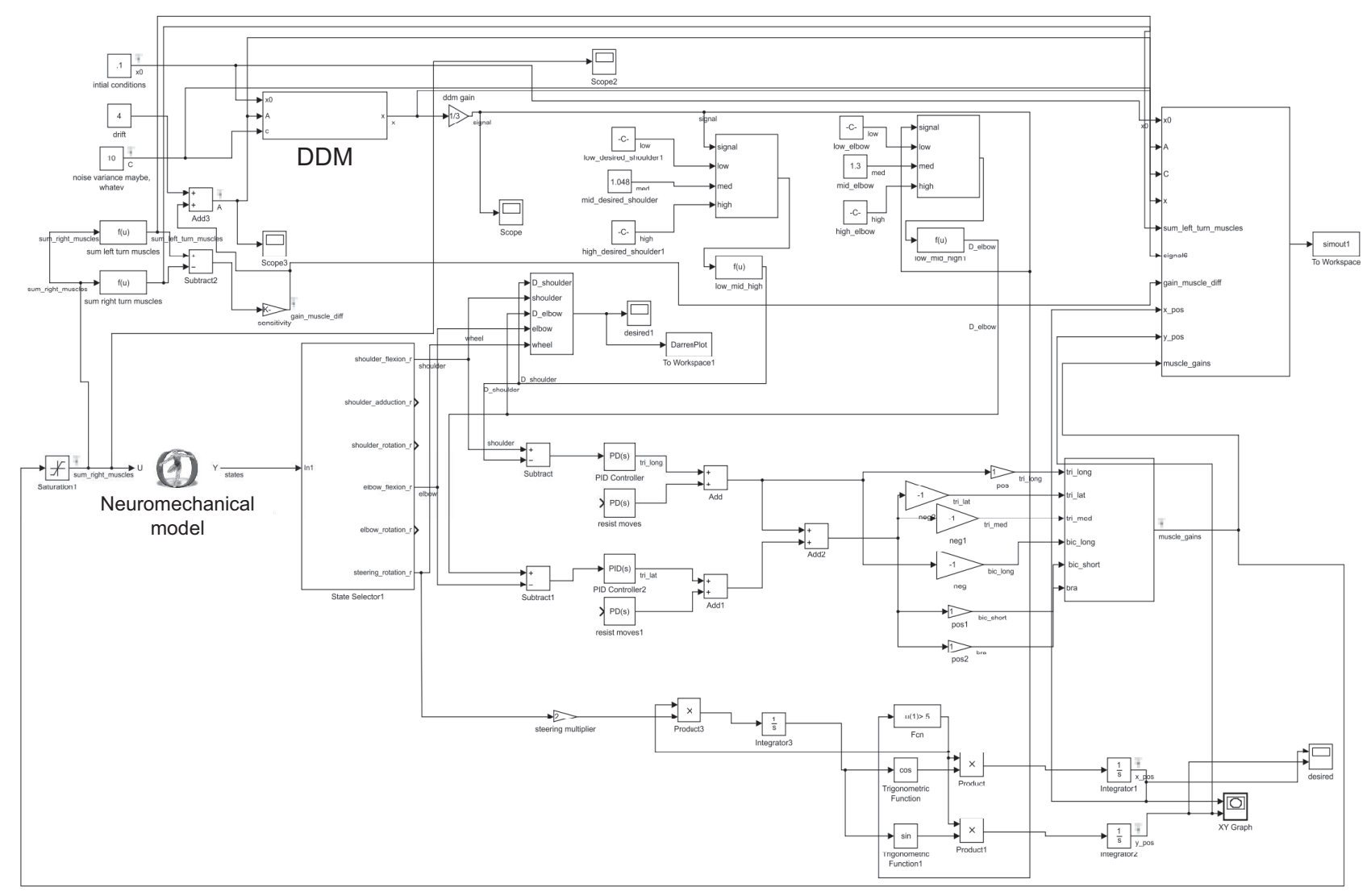

Fig. 7. Simulink model of coupled DDM and neuromechancial model. The driver makes a left turn based on the control signal from the DDM executed by the neuromechanical controller. The feedback from the neuromechanical model to the DDM consists of a contribution to the rate of evidence accumulation (drift rate) in the DDM.

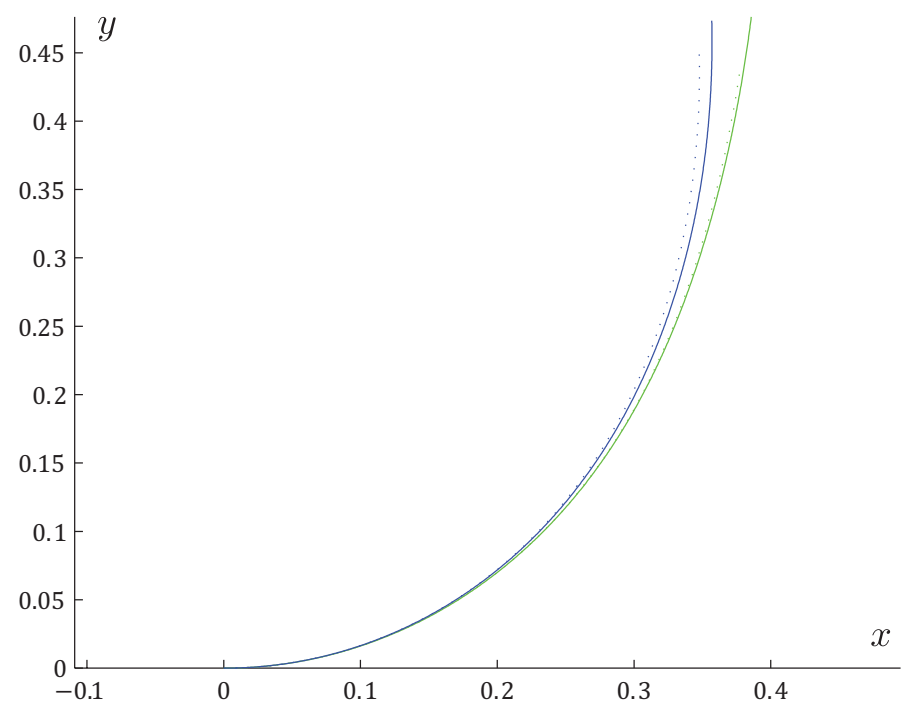

Fig. 10. Comparative trajectories over time. Trajectories for aggressive parameterizations are shown in green and for conservative parameterizations in blue. Strong coupling is indicated by solid lines and weak coupling by dashed lines, such that a green solid line indicates an aggressive driver with strong coupling.

\section{REFERENCES}

[1] L. P. Selen, M. N. Shadlen, and D. M. Wolpert, "Deliberation in the motor system: reflex gains track evolving evidence leading to a decision," The Journal of Neuroscience, vol. 32, no. 7, pp. 2276-2286, 2012.

[2] R. Bogacz, E. Brown, J. Moehlis, P. Holmes, and J. D. Cohen, "The physics of optimal decision making: a formal analysis of models of performance in two-alternative forced-choice tasks." Psychological Review, vol. 113, no. 4, p. 700, 2006.

[3] D. P. Hanes and J. D. Schall, "Neural control of voluntary movement initiation," Science, vol. 274, no. 5286, pp. 427-430, 1996.

[4] J. I. Gold and M. N. Shadlen, "The neural basis of decision making," Anпu. Rev. Neurosci., vol. 30, pp. 535-574, 2007.

[5] F. E. Zajac, "Critical reviews in biomedical engineering," in Muscle and tendon: properties, models, scaling, and application to biomechanics and motor control, J. R. Bourne, Ed. CRC Press, 1989, pp. 359-411.

[6] _ - "Muscle coordination of movement: a perspective," Journal of Biomechanics, vol. 26, pp. 109-124, 1993.

[7] O. Khatib, "A unified approach to motion and force control of robot manipulators: The operational space formulation." IEEE Journal of Robotics and Automation, vol. 3, no. 1, pp. 43-53, February 1987.

[8] - "Inertial properties in robotic manipulation: An object level framework," International Journal of Robotics Research, vol. 14, no. 1, pp. 19-36, February 1995.

[9] V. De Sapio, "An approach for goal-oriented neuromuscular control of digital humans in physics-based simulations," International Journal of Human Factors Modelling and Simulation, 2014, to appear.

[10] D. G. Thelen, F. C. Anderson, and S. L. Delp, "Generating dynamic 

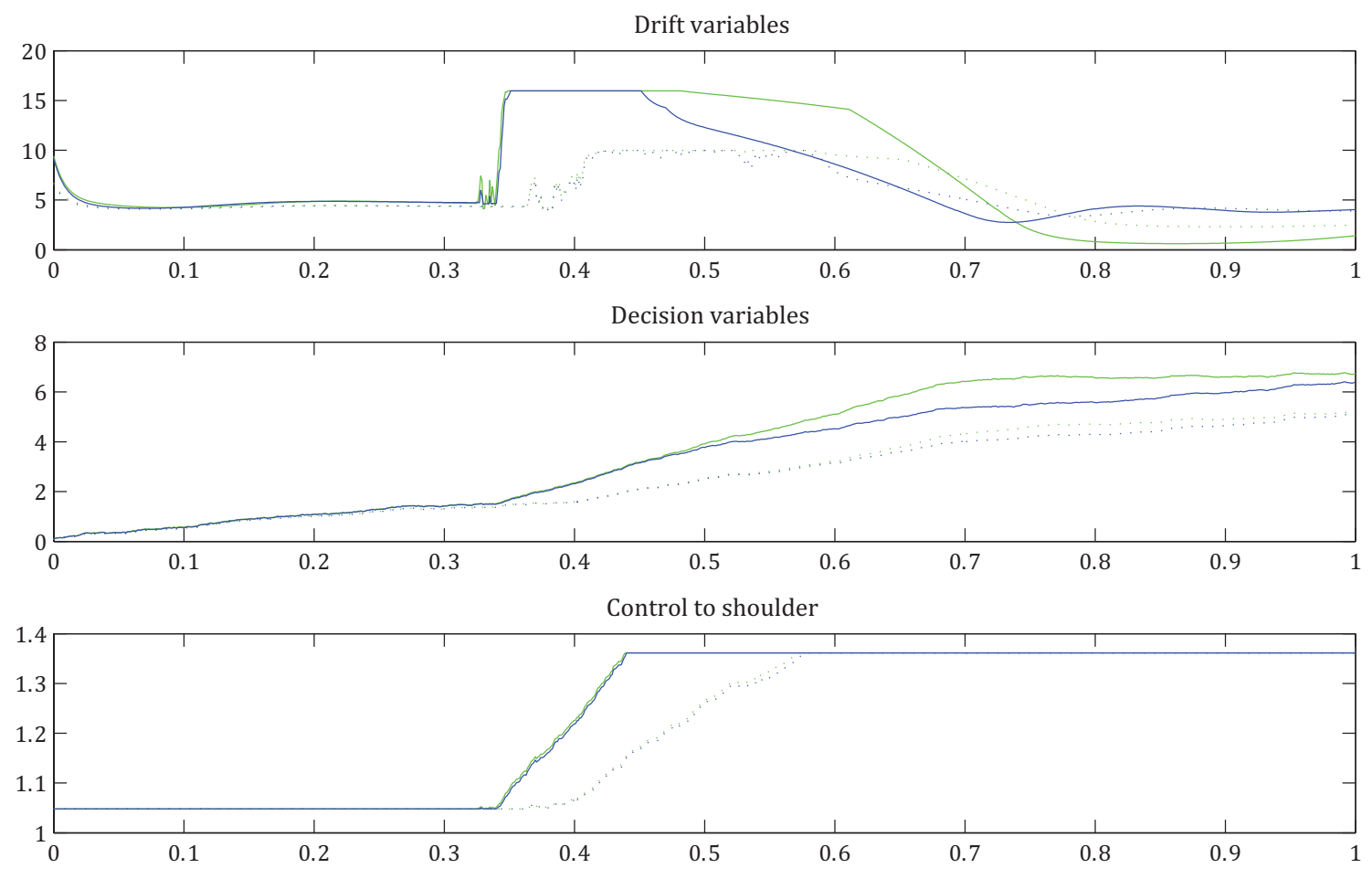

Control to elbow

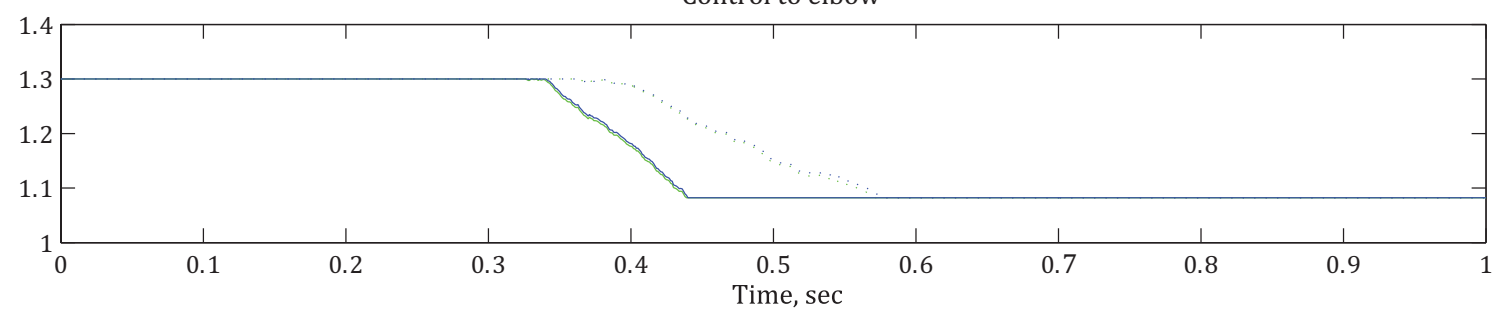

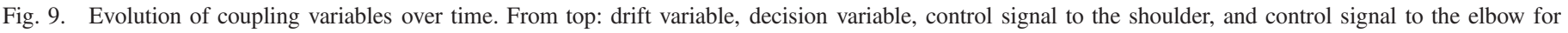

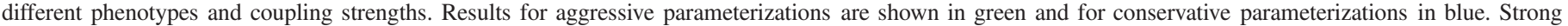
coupling is indicated by solid lines and weak coupling by dashed lines, such that a green solid line indicates an aggressive driver with strong coupling.

simulations of movement using computed muscle control," Journal of Biomechanics, vol. 36, pp. 321-328, 2003.

[11] D. G. Thelen and F. C. Anderson, "Using computed muscle control to generate forward dynamic simulations of human walking from experimental data," Journal of biomechanics, vol. 39, no. 6, p. 1107, 2006.

[12] M. Mansouri and J. A. Reinbolt, "A platform for dynamic simulation and control of movement based on opensim and matlab," Journal of biomechanics, vol. 45, no. 8, pp. 1517-1521, 2012.

[13] S. L. Delp, F. C. Anderson, A. S. Arnold, P. Loan, A. Habib, C. T. John, E. Guendelman, and D. G. Thelen, "Opensim: open-source software to create and analyze dynamic simulations of movement," Biomedical Engineering, IEEE Transactions on, vol. 54, no. 11, pp. 1940-1950, 2007. 